\title{
Development of Design and Analysis Tool for Switched Reluctance Drive System
}

\author{
Shoujun Song \& Weiguo Liu \\ School of Automation, Northwestern Polytechnical University \\ PO box 352, 127 Youyi Xilu, Xi'an, 710072, Shaanxi, China \\ Tel: 86-29-8843-1309 E-mail: songshoujun@hotmail.com
}

\begin{abstract}
Switched reluctance drive (SRD) system has been used in many applications. However, because of its inherent nonlinearity, the design and analysis of SRD system is relatively difficult. In this paper, the development of design and analysis tool for SRD system based on MATLAB/GUIDE is studied. Firstly, the steps for the development of graphical user interface (GUI) by MATLAB/GUIDE are summarized. Then, two tools, namely switched reluctance machine (SRM) design tool and converter thermal analysis tool, are presented as examples. The theoretical basis, structure and functions of both tools are presented. Results from each tool are given as well.
\end{abstract}

Keywords: Computer, MATLAB/GUIDE, Design, Analysis, Tool, Switched reluctance drive (SRD) system

\section{Introduction}

Many studies show that switched reluctance drive (SRD) system has many advantages, such as simple and rugged construction, flexible control, ability of high speed and temperature operation, low manufacturing cost and so on (W. Xu et al., 2009). All these advantages make the SRD system a prominent candidate for many kinds of applications (S. Song, 2009; X. D. Xue et al., 2008).

To increase the output power density, the switched reluctance machine (SRM) always operates in deep magnetic saturation. All the relevant characteristics of the machine, such as flux linkage and torque, are highly nonlinear functions of both rotor position and phase current (R. T. Naayagi et al., 2005). These nonlinearities make the design and analysis of the SRD system relatively difficult.

The design and analysis of the SRD system are related to many equations and data. What's more, there are lots of parameters that can affect the performances of the system seriously, so numerous combinations of these parameters need to be compared to obtain the optimal solution. To cope with heavy workload and increase the efficiency and accuracy of the design and analysis for SRD system, digital computers are used.

Graphical user interface (GUI) can greatly enhance the interactivity and efficiency of the design and analysis process. The workload of the engineers can be reduced significantly. What's more, the users do not need have much professional knowledge (L. Hong et al., 2009; G. D. Petropol-Serb et al., 2007). MATLAB has been widely used in many research fields. It has excellent capability for numerical solutions. In MATLAB, there is an integrated development environment for GUI named GUIDE which makes the development process of GUI easy and efficient (V. Ivanov et al., 2008; T. Hopp et al., 2008; M. Karacor et al., 2007).

In this paper, the development of design and analysis tool for SRD system based on MATLAB/GUIDE is studied. Two examples are given, namely SRM design tool and converter thermal analysis tool. The theoretical basis, structure and functions of both tools are presented. Results from each tool are given as well.

\section{Development of GUI by MATLAB/GUIDE}

MATLAB/GUIDE is an integrated development environment, which can help the users create the GUI conveniently. The steps for the development of GUI by MATLAB/GUIDE can be summarized as follows:

(1) Selection and arrangement of the components

In MATLAB/GUIDE, there are many useful components (controls), such as Slider, List Box and so on. Each component has its own function, for example, Slider can be used to change the value of a parameter within a certain range, and List Box can provide several options. According to the features and requirements of a certain application, users can select and arrange different components to build a practical and friendly GUI.

(2) Completion of the program for each component

In MATLAB, the program of GUI consists of an M-file and a figure file. They are connected by handles. Each component used in the figure file has a unique handle. After the creation of the figure file in step (1), the 
corresponding M-file can be generated automatically and users need to complete the callback function of each handle. The callback functions can be called according to the operations of users.

(3) Test and adjustment

After step (1) and (2), the created GUI need to be tested repeatedly and improved gradually in terms of accuracy, speed, reliability and interactivity.

(4) Save and release

Finally, the created GUI must be saved and released, so that it can run independently without the development environment and be installed in any computers.

The detailed information about MATLAB/GUIDE and the program can be easily found in many related books and papers.

\section{SRM design tool}

There are two traditional methods that can be used to design the SRM (T. J. E. Miller, 1993; J. Wu, 2000). The calculation procedures of these two methods are similar; both of them are based on the output equation. The only difference is the type of the output equation adopted. Eq. (1) and Eq. (2) show the output equations used in each method respectively.

$T=k D_{r}^{2} L_{s t k}$

where $T$ is rated torque, $k$ is $\mathrm{k}$ is the output coefficient proportional to the product of the electric and magnetic loadings, $D_{r}$ is the diameter of the rotor and $L_{s t k}$ is the length of the stack.

$$
P=\frac{A B k_{m} n D_{r}^{2} L_{s t k}}{6.1 \times 10^{11} k_{i}}
$$

where $P$ is rated power, $A$ is electric loading, $B$ is magnetic loading, $k_{m}$ is current coefficient of square wave, $n$ is rated speed and $k_{i}$ is current coefficient of the winding. In this paper, the typical values of $k_{m}$ and $k_{i}$ are adopted, namely $k_{m}=0.8$ and $k_{i}=0.5$.

In this paper, the methods based on Eq. (1) and Eq. (2) are called Method I and Method II respectively. It can be seen that Method I uses the output equation in terms of the torque, while Method II uses power.

According to Eq. (1) and Eq. (2), to determine $D_{r}$ and $L_{s t k}$ separately, it's necessary to select the length/diameter ratio $L_{s t k} / D_{r}$. Then, the simplest way to estimate $D_{s}$ from $D_{r}$ is through the typical ratio of $D_{r} / D_{s}$. Other dimensions of the machine can be easily obtained by some equations or experiences.

Fig. 1 shows the main interface of the SRM design tool. It can be seen that this interface includes five areas, namely Method selection, Performance parameters, Parameters selection, Typical values and Geometric dimensions \& Performance parameters. Firstly, users can choose any one method from those two methods mentioned above through Method selection. Then, the required performance parameters and related coefficients can be input through Performance parameters and Parameters selection. To help the users determine the values of some parameters, typical values are given in Typical values. Finally, the results can be obtained in Geometric dimensions \& Performance parameters by pushing the button marked by Calculate. An important function of this tool is that the affections of some selectable parameters on the dimensions and performance of the machine can be obtained by dragging the sliders after each selectable parameter. The values of the dimensions and performance will be changed dynamically along with the dragging. With this function, users can compare the results of different designs conveniently. Fig. 2 shows one design example.

\section{Converter thermal analysis tool}

The trend in power converter continues toward greater packing density, speed and high power dissipation. This technology trend resulted in increased operating temperatures, which have in turn lead to an increase of the failure rate and a reduction of the reliability. Thus, thermal analysis of the converter is playing an essential role in the design of high power systems such as vehicles, ships, aircrafts, etc. In order to ensure the heat dissipation ability of the converter, the thermal resistance of the selected heatsink should be considered carefully. Normally, there is a limitation on the thermal resistance value of the heatsink. In this paper, a tool is developed by MATLAB/GUIDE to implement thermal analysis of the asymmetric half-bridge converter (see Fig. 3) used in SRD system. By this tool, the limitation on the thermal resistance value of the heatsink can be obtained conveniently.

Fig. 4 shows the static thermal model of a three-phase asymmetric half-bridge converter, where $\mathrm{P}_{\text {total }}$ is the total 
power losses of one IGBT, RthJC is the thermal resistance between the junction and the case, RthCH is the thermal resistance between the case and the heatsink, RthHA is the thermal resistance of the heatsink and $\mathrm{T}_{\mathrm{a}}$ is ambient temperature. In this paper, all the power components are assumed to be fixed on one heatsink. To simplify the analysis, the diodes are omitted, so only six IGBTs need to be considered.

As we know that the temperature rise is caused by the power losses. So, before the thermal analysis of the converter, the power losses in it must be calculated firstly. The power losses in IGBT occur during conduction (conduction losses) and switching transients (switching losses).

The conduction losses of the IGBT can be calculated in a straightforward manner as the product of the voltage across and the current through the IGBT. Thus average conduction loss in the IGBT can be expressed as,

$$
P_{c o n}=\frac{1}{T} \int_{0}^{T} v_{c e} i_{c} d t=\frac{1}{T} \int_{0}^{T}\left(V_{0}+r i_{c}\right) i_{c} d t=\frac{V_{0}}{T} \int_{0}^{T} i_{c} d t+\frac{r}{T} \int_{0}^{T} i_{c}^{2} d t=V_{0} I_{\text {mean }}+r I_{r m s}^{2}
$$

where, $T$ is the conduction period, $v_{c e}$ and $i_{c}$ are the instantaneous values of voltage and current respectively that occurs during conduction, $I_{\text {mean }}$ and $I_{r m s}$ are the average and rms values of IGBT current respectively. $V_{0}$ and $r$ are dc portion of saturated voltage and equivalent resistance of saturated IGBT respectively which can be obtained by the linearization of on-state IV characteristics of the IGBT.

The switching losses are due to non-ideal switching process of the IGBT. It is the sum of two parts: turn-on switching losses and turn-off switching losses. It can be easily calculated by

$$
P_{s w}=f_{s w} \cdot\left(E_{o n}+E_{o f f}\right)
$$

where $f_{s w}$ is the switching frequency. $E_{o n}$ and $E_{\text {off }}$ are turn-on and turn-off energy losses in the IGBT respectively.

The total power losses of one IGBT can be obtained by

$$
P_{\text {total }}=P_{c o n}+P_{s w}
$$

According to Fig. 4, to ensure the junction temperature of the IGBT do not exceed the maximum value, the following inequation must be satisfied.

$$
P_{\text {total }}(\text { RthJC }+R \text { th } C H+6 \text { RthHA }) \leq T J m a x-T a
$$

where TJmax is the limitation of junction temperature.

From Eq. (6), the limitation value of the heatsink thermal resistance can be obtained as follows,

$$
R \operatorname{thH} A=\frac{1}{6}\left(\frac{\text { TJmax }-T a}{P_{\text {total }}}-\text { RthJC }- \text { RthCH }\right)
$$

Fig. 5 shows the main interface of the converter thermal analysis tool. It can be seen that this interface includes three areas, namely IGBT parameters, Rating parameters and Output parameters. Firstly, some parameters of the IGBT related to the thermal analysis can be input through IGBT parameters. Then, some parameters of the system can be input through Rating parameters. Finally, the results include conduction losses, switching losses, total losses and the limitation value of the heatsink thermal resistance can be obtained in Output parameters by pushing the button marked by Calculate. Fig. 6 shows one analysis example.

\section{Conclusion}

In this paper, the development of design and analysis tool for SRD system based on MATLAB/GUIDE is studied. Firstly, the steps for the development of GUI by MATLAB/GUIDE are summarized. Then, two examples are given, namely SRM design tool and converter thermal analysis tool. The theoretical basis, structure and functions of both tools are presented. The SRM design tool provides two traditional design methods. The geometric dimensions and performance parameters can be got quickly. What's more, the affections of some selectable parameters on the dimensions and performance of the machine can be obtained conveniently. The converter thermal analysis tool can get the conduction losses, switching losses, total losses of the IGBT and the limitation value of the heatsink thermal resistance quickly.

\section{References}

G. D. Petropol-Serb, I. Petropol-Serb, A. Campeanu and A. Petrisor. (2007). Using GUI of Matlab to create a virtual laboratory to study an induction machine. Proceedings of International Conference on "Computer as a Tool", Warsaw, Poland, Sept., pp. 2355-2360.

J. Wu. (2000). Design and application of switched reluctance machine. Beijing, China: China Machine Press.

L. Hong, J. Cai and B. Cui. (2009). GUI Based Simulation Platform for Inverter Drive System under Fault 
Conditions. Proceedings of Second International Symposium on Knowledge Acquisition and Modeling, Wuhan, China, Dec., pp. 131-134.

M. Karacor and F. E. Kuyumcu. (2007). Matlab GUI based SRM design program. Proceedings of International Aegean Conference on Electrical Machines and Power Electronics, Bodrum, Turkey, Sept., pp. 846-849.

R. T. Naayagi and V. Kamaraj. (2005). A Comparative Study of Shape Optimization of SRM using Genetic Algorithm and Simulated. Proceedings of IEEE Indicon 2005 Conference, Chennai, India, Dec., pp. 596-599.

S. Song. (2009). Detailed design of a $30 \mathrm{~kW}$ switched reluctance starter/generator system used in more/all electric aircraft. Aachen, Germany: Shaker Verlag.

T. Hopp, G. F. Schwarzenberg, M. Zapf and N. V. Ruiter. (2008). A MATLAB GUI for the Analysis and Exploration of Signal and Image Data of an Ultrasound Computer Tomograph. Proceedings of First International Conference on Advances in Computer-Human Interaction, Sainte Luce, Martinique, Feb., pp. 53-58.

T. J. E. Miller. (1993). Switched reluctance motors and their control. Oxford, UK: Clarendon Press.

V. Ivanov, S. Ivanov and M. Brojboiu. (2008). Matlab graphical user interface for system with Dallas microcontroller. Proceedings of International Symposium on Power Electronics, Electrical Drives, Automation and Motion, Ischia, Italy, Jun., pp. 502-507.

W. Xu, J. Zhu, Y. Guo, S. Wang, Y. Wang and Z. Shi. (2009). Survey on electrical machines in electrical vehicles. Proceedings of International Conference on Applied Superconductivity and Electromagnetic Devices, Chengdu, China, Sept., pp. 167-170.

X. D. Xue, K. W. E. Cheng and N. C. Cheung. (2008). Selection of Electric Motor Drives for Electric Vehicles. Proceedings of 2008 Australasian Universities Power Engineering Conference, Sydney, NSW, Dec., pp. 1-6.

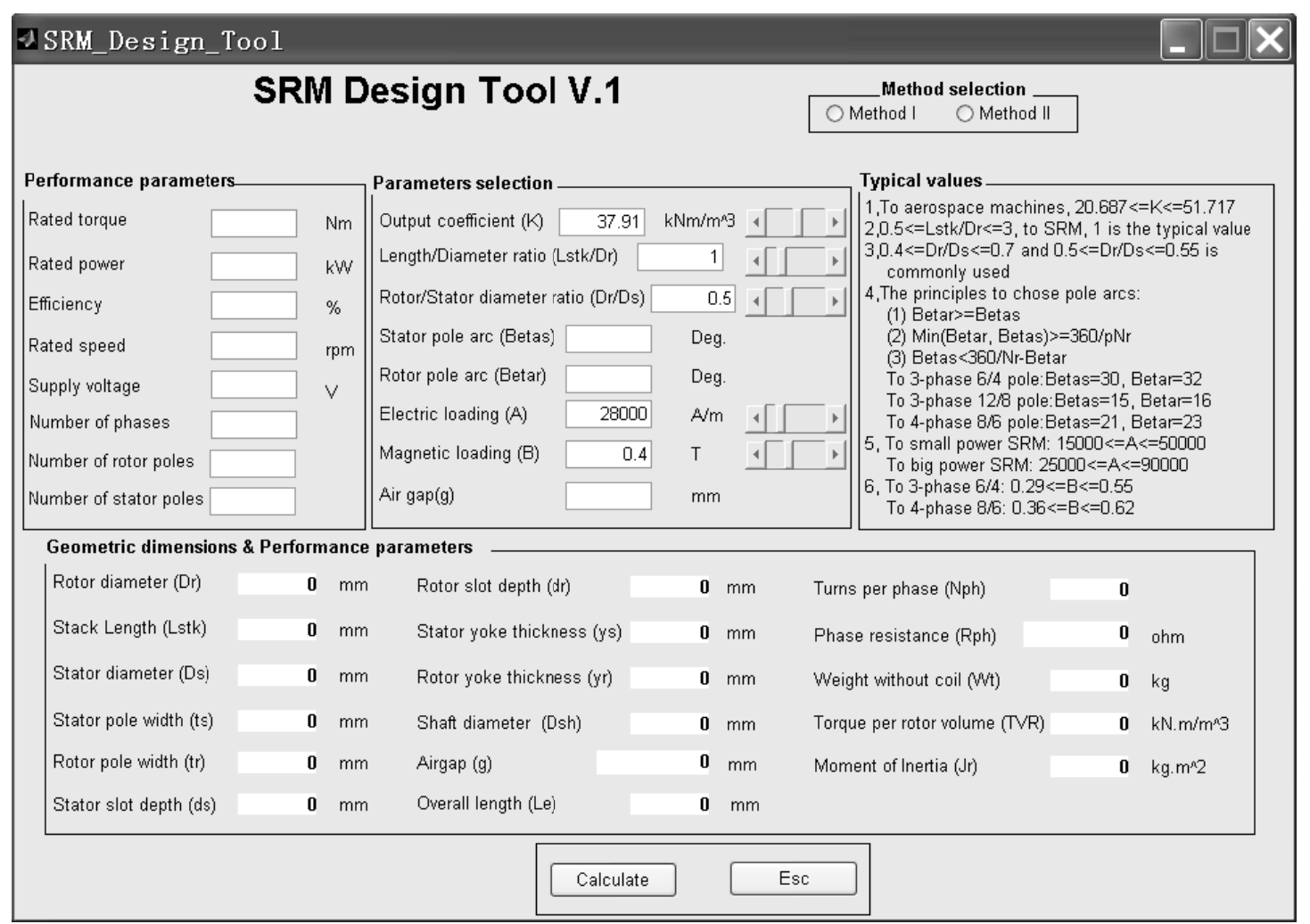

Figure 1. Main interface of SRM design tool 


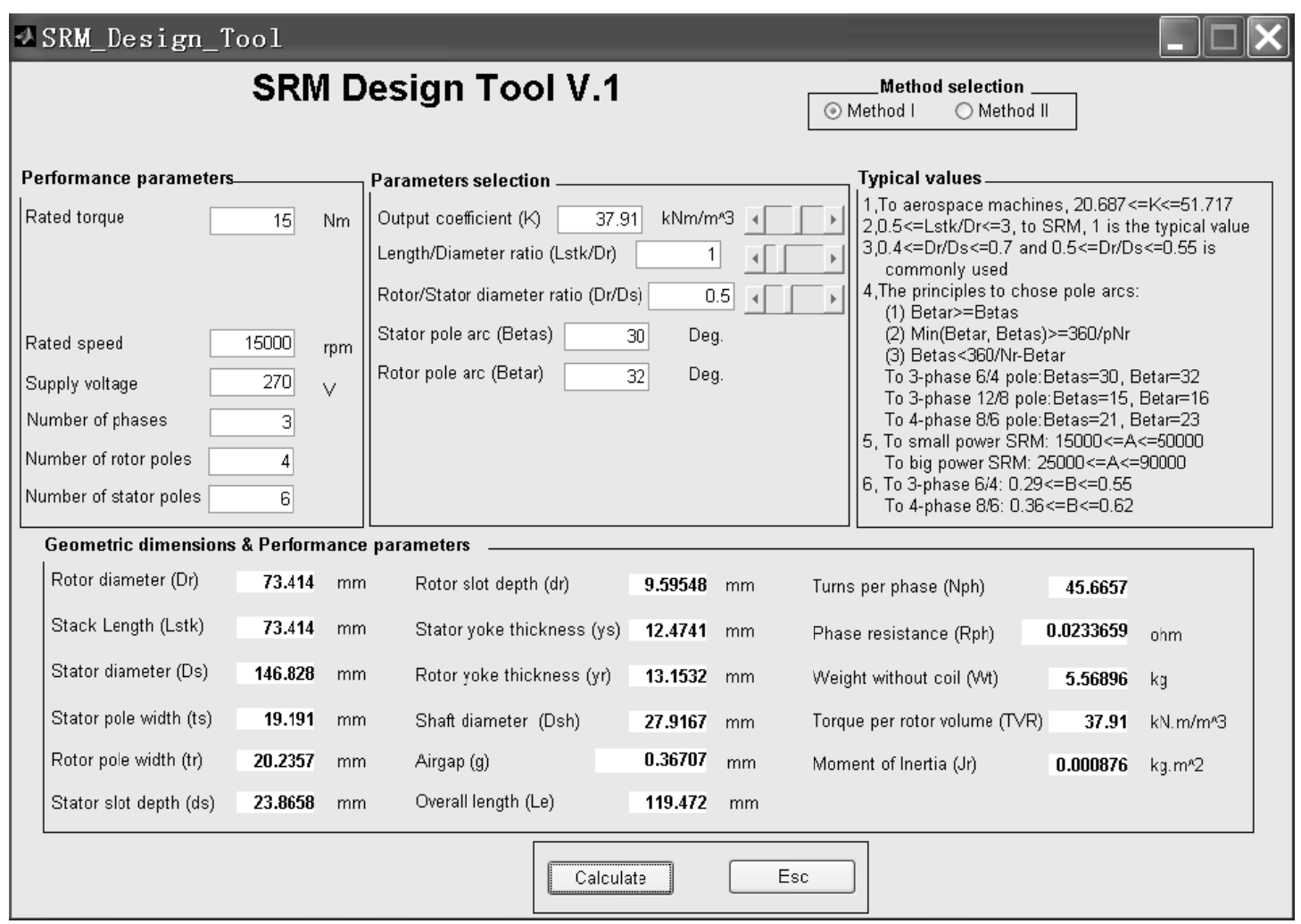

Figure 2. One design example by SRM design tool

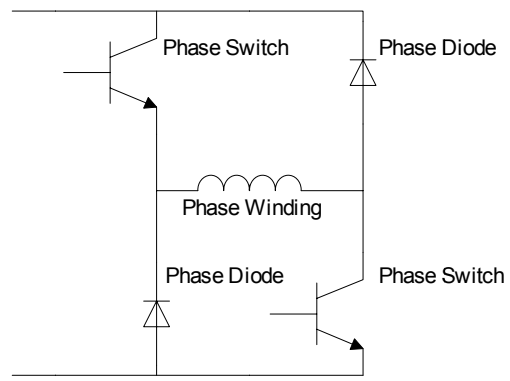

Figure 3. Asymmetric half-bridge converter circuit (one phase)

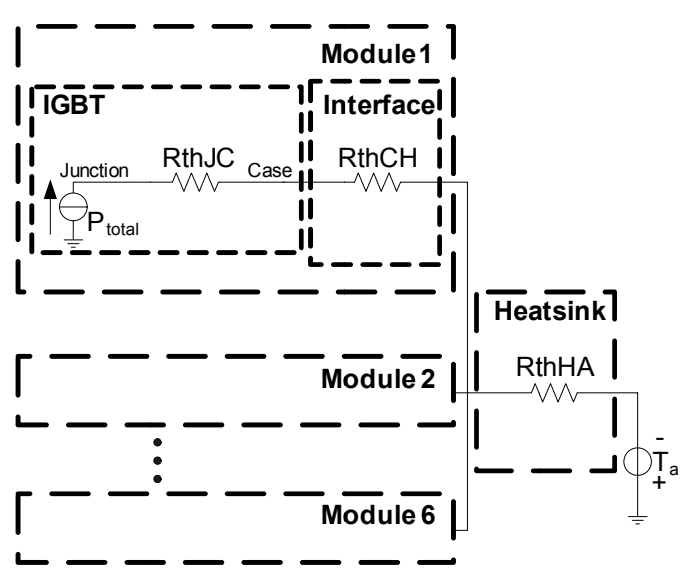

Figure 4. Thermal model of a three-phase asymmetric half-bridge converter 


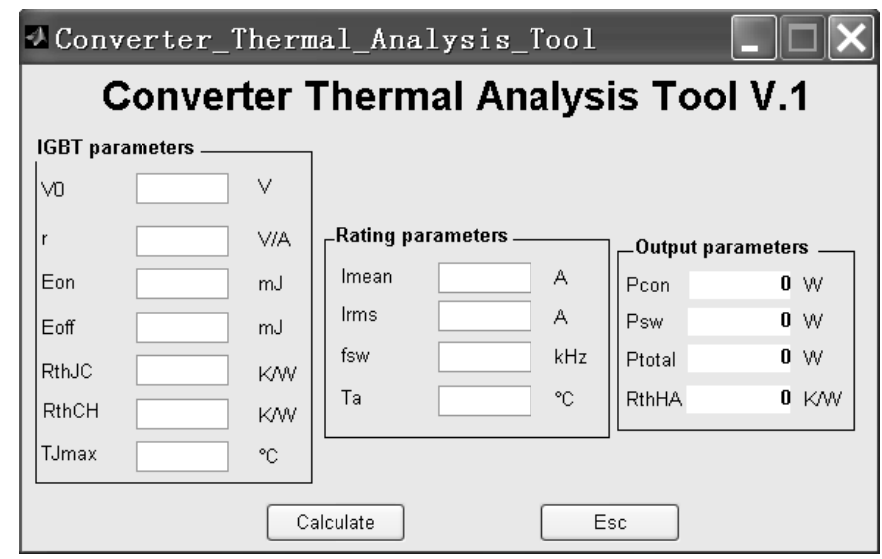

Figure 5. Main interface of converter thermal analysis tool

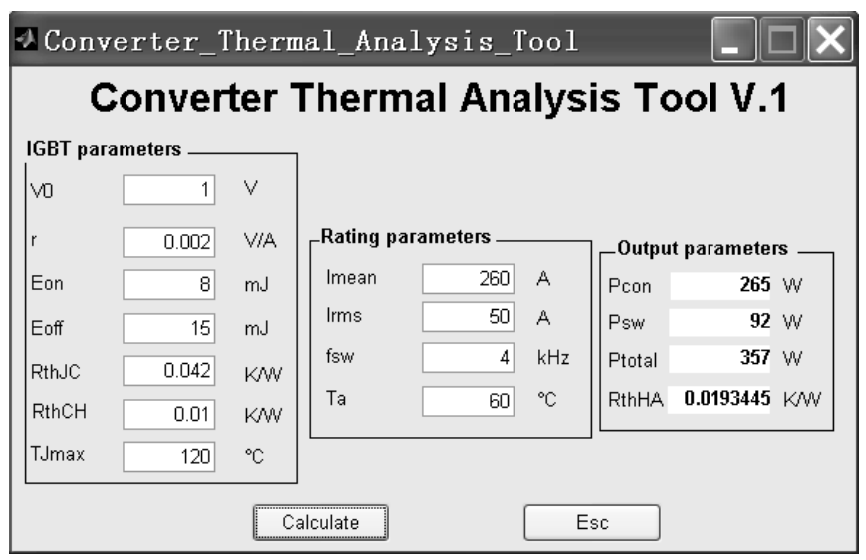

Figure 6 . One design example by converter thermal analysis tool 